\title{
Atuação do farmacêutico clínico frente à COVID-19 em um hospital público da região amazônica
}

\author{
Performance of the clinical pharmacist against COVID-19 in a public hospital in the \\ amazon region
}

\section{Actuación del farmacéutico clínico frente a COVID-19 en un hospital público de la región amazónica}

Welliny Mariana Posse Fuzari ${ }^{1 *}$, Elissa Gonçalves Oliveira e Silva ${ }^{1}$, Roberta Grain Barreto Cardoso ${ }^{1}$, Simone Rosária Soares de Moraes Cunha ${ }^{1}$, Danielle Maesima Saito ${ }^{1}$, Valcione da Silva Gretzler ${ }^{1}$, Thais Dias Zumack¹.

\section{RESUMO}

Objetivo: Relatar a implantação de uma farmácia satélite, bem como a elaboração de protocolos, ações gerenciais e farmacêutico-clínicas no enfrentamento da pandemia COVID-19, em um hospital público de alta complexidade da região amazônica, referência em atendimento a pacientes acometidos pela COVID-19. Relato de experiência: Este relato concerne sobre as atividades clínicas e gerenciais desenvolvidas pelos farmacêuticos de um hospital público da região amazônica durante a pandemia causada pelo SARS-CoV-2, ações que se fizeram notáveis e de suma importância, baseadas na criação de protocolos clínicos, estruturação de setores, adequação de rotinas e comunicação efetiva com a equipe multiprofissional, buscando atualização contínua e adaptação aos desafios que foram surgindo. Considerações finais: A pandemia pelo vírus SARS-CoV-2, trouxe diversos desafios e expôs as fragilidades na assistência em saúde, por ser algo sem precedentes, entretanto também reforçou a importância do farmacêutico na equipe multidisciplinar para melhora da assistência prestada através de atividades clínicas e gerenciais.

Palavras-chave: COVID-19, Atenção farmacêutica, Assistência farmacêutica, Serviço de farmácia hospitalar.

\begin{abstract}
Objective: To report the implementation of satellite Pharmacy as well as the devolpmente of protocols, managerial and pharmaceutical-clinical actions to deal with the COVID-19 pandemic in a highly complex public hospital in the Amazon region, a reference in the care of patients affected by COVID-19. Experience report: This report concerns the clinical and management activities carried out by pharmacists at a public hospital in the Amazon region during the pandemic caused by SARS-CoV-2, actions that became notable and extremely important, based on the creation of clinical protocols, structuring of sectors, restructruring of routines and effective communication with the multiprofessional team seeking continuos updating to the challenges that were faced. Final considerations: The unprecedentend pandemic caused by the SARS-CoV-2 virus, brought up several challenges and weaknesses in health care, however it also reinforced the importance of the pharmacist in the multidisciplinary team to improve the care provided.
\end{abstract}

Keywords: COVID-19, Pharmaceutical attention, Pharmaceutical assistance, Hospital pharmacy service.

\section{RESUMEN}

Objetivo: Informar la implementación de la Farmacia satélite, así como el desarrollo de protocolos, acciones gerenciales y farmacêutico-clínicas para enfrentar la pandemia de COVID-19 en un hospital público de alta complejidad en la región amazónica, referencia em la atención de pacientes afectados por COVID-19. Informe de experiencia: Este informe se refiere a las actividades clínicas y de gestión desarrolhadas por famacéuticos em um hospital público de la región amazónica durante la pandemia causada por el SARS-Cov-2, acciones que cobraron relevancia y suma importancia a partir de la elaboración de protocolos clínicos, estructuración de sectores, adecuanción de rutinas y comunicación efectiva con el equipo multiprofesional buscando la

\footnotetext{
${ }^{1}$ Hospital Regional de Cacoal (HRC), Cacoal - RO. *E-mail: wellinypossefuzari@gmail.com
} 
continua actualización y adaptación a los retos que se han presentado. Consideraciones finales: La pandemia provocada por el vírus SARS-CoV-2, trajo varios desafios y debilidades em la atención de la salud, por ser algo inédito, sin embargo también reforzó la importancia del farmacêutico em el equipo multidisciplinario para mejorar la atención brindada.

Palabras clave: COVID-19, Atención farmacéutica, Asistencia farmacéutica, Servicio de farmacia hospitalaria.

\section{INTRODUÇÃO}

Em dezembro de 2019, surgiu na China, mais especificamente na cidade de Wuhan, um vírus da família Corona, que viria afetar a saúde global. Do oriente teve uma rápida propagação alcançando todos os continentes, sendo que o primeiro caso no Brasil foi identificado ao final de janeiro de 2020. Os pacientes apresentavam sinais clínicos característicos de pneumonia atípica, como febre, tosse, dispneia e mialgia ou fadiga (BARLOW A, et al., 2020).

O vírus responsável por esta pneumonia foi posteriormente identificado como um novo Coronavírus, do gênero dos betacoronavírus, denominado SARS-CoV-2, pela sua semelhança genômica com o vírus responsável por causar a SARS (Síndrome da Angústia Respiratória Grave) no ano de 2003 e o Coronavírus responsável por causar a MERS (Síndrome Respiratória do Oriente Médio) no ano de 2012. Ambas as síndromes com sinais clínicos inespecíficos, tendo como característica principal febre e sintomas respiratórios (WU Y, et al., 2020).

A doença causada pela nova cepa do coronavírus, denominada como 2019-nCoV (novo coronavírus), ficou conhecida mundialmente pela sigla COVID-19, podendo se manifestar de forma leve, moderada ou grave, com alterações desde sintomas leves até insuficiência respiratória grave, além do comprometimento de outros sistemas orgânicos como o renal, cardiovascular e neurológico, exigindo assim a admissão em unidade de terapia intensiva (BARLOW A, et al., 2020).

Em 11 de março de 2020, a COVID-19 foi oficialmente declarada como pandemia pela OMS (Organização Mundial de Saúde). Sua transmissão entre humanos ocorre por meio de gotículas e aerossóis respiratórios, liberados durante a tosse ou espirro, ou ainda através do contato com superfícies e objetos infectados, quando tocados e levados ao rosto, nariz ou boca. As medidas preventivas sugeridas são de simples realização, sendo aplicadas mundialmente: isolamento social, lavagem correta das mãos com água e sabão, uso do álcool em gel 70\%, limpeza de superfícies com álcool a 70\% e o uso de máscaras (SILVA LMC e ARAUJO $\mathrm{JL}, 2020$ ).

Devido ao fato de ainda não existir uma vacina, nem uma terapia medicamentosa específica para o tratamento da COVID-19, os farmacêuticos se tornaram ferramentas essenciais para a disseminação do Uso Racional de Terapias Medicamentosas, tanto a nível ambulatorial quanto hospitalar, através do desenvolvimento da farmácia clínica (SILVA LMC e ARAUJO JL, 2020).

A resolução $n^{\circ} 585$, de 29 de agosto de 2013 , define farmácia clínica como a área da farmácia voltada à ciência e à prática do uso racional de medicamentos, na qual os farmacêuticos prestam cuidado ao paciente, de forma a otimizar a farmacoterapia, promover saúde, bem-estar e prevenir doenças. Em se tratando de âmbito hospitalar, as atividades clínicas dos farmacêuticos se norteiam por meio da avaliação das prescrições médicas, considerando interações medicamentosas, falhas de aprazamento, compatibilidades de vias de administração, farmacoterapia adequada ao diagnóstico do paciente, entre outros parâmetros de avaliação (BERNARDI EAT, et al., 2014). O desenvolvimento destas atividades permite a identificação precoce dos Problemas Relacionados aos Medicamentos (PRM) e garante que o paciente receba uma farmacoterapia adequada e segura (SILVA BC e OLIVEIRA JV, 2012; PELENTIR M, et al., 2015).

A abordagem multidisciplinar no atendimento de pacientes acometidos pela COVID-19 ficou em evidência neste período pandêmico. O acometimento de vários órgãos como pulmão, coração, rins e outros, exigiu participação de vários profissionais respeitando as limitações éticas de cada profissão, além de mudanças 
constantes nas condutas terapêuticas, sejam elas medicamentosas, fisioterapêuticas ou outras (VALLE MCD, et al., 2020). Esse comprometimento múltiplo requer na maioria das vezes internação em Unidades de Terapia Intensiva (UTI), essa internação torna mais frequente a necessidade de alterações constantes de terapias farmacológicas, aumentando assim os riscos associados a internação (SOCORRO FHOS, et al., 2020).

A pluralidade de medicamentos, vias de acesso, formas farmacêuticas, aumentam o risco de problemas relacionados a medicamentos e Reações Adversas a Medicamentos (RAM). A participação do farmacêutico clínico durante todas as etapas que envolvem os medicamentos é de suma importância para a redução de erros (PILAU R, et al., 2014). Sugestões de alterações nas prescrições, validação correta da dispensação com análise de vias de administração, forma farmacêutica, doses terapêuticas, informações constantes as equipes de enfermagem, permitindo assim a otimização e maior segurança da terapia farmacológica (CARDINAL L e FERNANDES C, 2014).

A realização de serviços clínicos farmacêuticos é algo novo na maioria das instituições hospitalares, entretanto foi um serviço muito requisitado durante a pandemia da COVID-19, em razão da crise enfrentada pela indústria farmacêutica e pelas empresas fabricantes de materiais médico-hospitalares, fato este desencadeado pela falta de matéria-prima para a fabricação de medicamentos, descontinuidade de produção, principalmente de analgésicos e sedativos, provocando assim significativo aumento dos preços, além do desabastecimento em decorrência do elevado consumo, restrições de fronteiras, fechamento das indústrias fabricantes devido redução das equipes, em todo o mundo (CASSANO AO e AREDA AC, 2020).

Diante do exposto este relato tem como objetivo demonstrar a implantação de uma farmácia satélite, com espaço e localização estratégica para atendimento das unidades de terapia intensiva responsáveis pelo tratamento dos pacientes acometidos pela COVID-19, bem como a elaboração de protocolos, ações gerenciais e farmacêutico-clínicas no enfrentamento da pandemia ocasionada pela COVID-19 em um hospital público de alta complexidade da região amazônica, referência em atendimento a estes pacientes.

\section{RELATO DA EXPERIÊNCIA}

O período de pandemia pela COVID-19 decretada em março de 2020, pela OMS, exigiu de todas as categorias profissionais, não apenas da área da saúde, mas de toda a sociedade, adaptação e inovação nas metodologias de trabalho.

No que tange ao hospital em questão, houve a necessidade de adaptação estrutural para atendimento dos pacientes infectados por SARS-Cov-2. Primordialmente foi criada a Unidade 1 COVID, da qual a equipe do serviço de farmácia hospitalar se fez presente, padronizando e criando armário de emergência, maleta de emergência com drogas sujeitas a controle especial, como prevê a portaria no 344 de 12 de maio de 1998 , carrinho de emergência e carinho de parada cardiorrespiratória, buscando adaptações conforme a infraestrutura das enfermarias COVID. As padronizações foram feitas com base nas recomendações da Sociedade Brasileira de Farmácia Hospitalar (SBRAHF) e nas recomendações da Associação de Medicina Intensiva Brasileira (AMIB), além de protocolos já estabelecidos nas UTI's do hospital e de protocolos de instituições de saúde com reconhecimento nacional.

Com o aumento do número de pacientes críticos exigindo internação em UTI, consequentemente houve a expansão dos números de leitos, aumentando a ala destinada ao atendimento de casos de COVID e elevando a demanda de consumo de medicamentos e materiais médico-hospitalares, sendo assim realizada a implantação de uma farmácia satélite. No que se refere ao setor farmacêutico deste hospital, visando à melhoria na assistência prestada, fez-se necessário a criação e adaptação de uma farmácia satélite, implantada para atendimento específico de pacientes da COVID-19, foco deste relato. Um espaço com localização estratégica, de fácil acesso às unidades COVID, destinado à dispensação e ao fornecimento de medicamentos e materiais médico-hospitalares aos setores.

Essa implantação ocorreu por meio da iniciativa das farmacêuticas residentes em terapia intensiva, com total apoio da gerência de farmácia. Sendo estabelecido novo fluxo para atendimento das prescrições, visando a adaptação ao horário de funcionamento da farmácia satélite, das 07h00min às 19h00min diariamente. Este 
novo setor também proporcionou uma maior interação com a equipe multiprofissional, permitindo uma gestão efetiva da farmácia clínica por parte da equipe farmacêutica da residência multiprofissional em cuidados intensivos.

A dispensação de medicamentos nesta unidade é realizada de forma individualizada a cada paciente para atender um período de vinte e quatro horas, serviço este realizado pelos farmacêuticos residentes e plantonistas. Visando atender as recomendações da Sociedade Brasileira de Farmácia Hospitalar (SBRAHF), no que se refere ao cuidado e avaliação farmacêutica, é recomendada a análise das prescrições, a qual foi facilitada pela abertura da farmácia satélite, permitindo a execução de intervenções farmacêuticas relacionadas às posologias, vias de administração, compatibilidades, formas farmacêuticas disponíveis na instituição, interações medicamentosas, entre outras intervenções que minimizaram os problemas relacionados à farmacoterapia.

Para a implantação da farmácia satélite também foram estabelecidas a instalação e padronização de armário de emergência, composto por estoque mínimo das principais medicações usadas em pacientes críticos, estoque de materiais médicos hospitalares, novo fluxo de atendimento e abastecimento, levando a uma provável diminuição da propagação de contaminação pelos outros setores do hospital e facilitando o acesso a informações sobre terapias medicamentosas para toda a equipe.

Diante da crise nos estoques de materiais médico hospitalares e principalmente medicações, foi necessário a implementação de protocolos clínicos de uso de drogas vasoativas, sedativos, hipnóticos, bloqueadores neuromusculares e analgésicos. Estabelecendo suas diluições, concentrações, volumes máximos e mínimos para infusão contínua, buscando o uso seguro em limiares terapêuticos, permitindo a otimização dos serviços desempenhados pela equipe, e garantindo a melhor assistência possível, além de padronizar as condutas e alinhamento da comunicação entre a equipe multiprofissional, permitindo também o uso racional e gerenciamento dos estoques que se encontravam baixos.

Um dos problemas enfrentados pela equipe da COVID foi a escassez de sedativos e analgésicos utilizados na intubação e na manutenção de pacientes, em especial o midazolam e a fentanila, assim, se fez necessária a utilização de protocolos alternativos que realizam a substituição dessas drogas, as bombas de infusão multimodais, com drogas como Clonidina, dexmedetomidine e licocaina, drogas estas já utilizadas em práticas cirúrgicas, foram utilizadas como terapias alternativas para estes pacientes em tempos de crises, assim como o uso de infusão continua de morfina se tornou uma realidade, devido a falta do analgésico fentanila.

A necessidade de um alinhamento de condutas clínicas exigiu a elaboração de protocolos, além da utilização de terapias infusionais contínuas para pacientes críticos, foram também estabelecidos informativos para uso de hidroxicloroquina e azitromicina tanto em pacientes críticos como clínicos, enquanto suas recomendações científicas eram válidas.

A elaboração deste protocolo foi conforme as Diretrizes Terapêuticas para Diagnóstico e Tratamento da COVID-19 emitida em 6 de abril de 2020 pelo Ministério da Saúde, estabelecendo a dose recomendada de hidroxicloroquina em pacientes hospitalizados, sendo 1 comprimido de $400 \mathrm{mg}$ 2x/dia no primeiro dia (800 mg de dose de ataque), seguido de 1 comprimido $400 \mathrm{mg} \mathrm{1x/dia} \mathrm{no} \mathrm{segundo,} \mathrm{terceiro,} \mathrm{quarto} \mathrm{e} \mathrm{quinto} \mathrm{dia} \mathrm{(400}$ $\mathrm{mg} / \mathrm{dia}$ ), da mesma forma que o uso de hidroxicloroquina foi descontinuado após corroborações cientificas afirmarem que ela teve pouco ou nenhum efeito em pacientes hospitalizados com Covid-19, como mostra o estudo Repurposed Antiviral Drugs for Covid-19 - Interim WHO Solidarity Trial Results publicado pelo The new england journal o f medicine (CONSORTIUM WST, 2020).

\section{DISCUSSÃO}

A tomada de decisões diante a uma situação inusitada sem respaldo científico, se tornou um desafio neste ano de 2020, para os profissionais da linha de frente no enfrentamento à COVID-19. A necessidade da criação de protocolos clínicos e padronizações de estoques mínimos de medicamentos e materiais se fez necessária em todas as alas COVID. 
Como mostra Brasil (2020); terapias medicamentosas para a COVID-19, foram baseadas em evidências terapêuticas já utilizadas em outros vírus do grupo dos betacoronavírus, podendo ser alteradas conforme a estruturação de novos estudos, como assim se fez com o uso da cloroquina e da hidroxicloroquina. A otimização dos serviços desenvolvidos pelas equipes de farmácia hospitalar, através da implantação de farmácias satélites e a descentralização das atividades desenvolvidas, ficou evidenciado como uma ação acertiva (MCCONACHIE S, et al.,2020).

A implantação de uma farmácia satélite assim como a realização de padronizações permitiu uma redução na circulação da equipe da unidade COVID, pelas outras alas hospitalares, ocasionando uma provável redução na propagação do vírus, redução de estoques desnecessários, assim como a acessibilidades as drogas de urgência e emergência, condutas que facilitaram as adaptações as novas rotinas de trabalho das equipes, estes benefícios também foram identificados no estudo de Dantas FA (2019) com a implantação de farmácia satélite em um pronto socorro.

O alinhamento de condutas e adequação conforme o aparecimento de evidências cientificas é algo contínuo, visto que o manejo clínico da COVID-19 é fato novo, sendo necessária a capacitação e a atualização contínuas sobre as novas e usuais terapias para o grupo dos betacoronavirus.

Quando tratamos de pacientes internados em unidades de terapia intensiva, deparamo-nos com casos críticos e polimedicamentosos, fato este agrava os riscos provenientes desta internação, pois aumenta a possibilidade de interações medicamentosas, retardando a evolução clínica do paciente, situação essa já prejudicada pelo uso prolongado de sedativos e bloqueadores neuromusculares, medicações de extrema importância para esta doença, fazendo-se notar mais uma vez o importante papel da equipe multidisciplinar, não somente no tratamento, mas também na reabilitação destes pacientes, esta colaboração da equipe multiprofissional também ficou evidenciada no estudo de Gil MG e Amo CV (2020).

A revisão de Monteiro FCC, et al., (2020); reforça que cabe ao farmacêutico e à equipe multiprofissional monitorar o uso destas medicações, por serem terapias novas com poucos indícios científicos, podendo causar agravos ou retardar a evolução clínica dos pacientes acometidos pela COVID-19. Assim como Llamazares CMF e Briz EL (2020); afirma a contribuição da elaboração de protocolos clínicos no norteamento de condutas da equipe multiprofissional além de favorecer o desenvolvimento das atividades clínicas farmacêuticas.

O cuidado com a estabilidade das drogas, seja após diluição ou reconstituição, foi fundamental para a economia e o aproveitamento de recursos escassos, na forma como conduzimos a farmácia satélite. O papel executado pelas farmacêuticas residentes em orientar nessas condutas foi de grande valia para minimizar a utilização dos poucos medicamentos.

Os cuidados farmacêuticos acerca dos pacientes acometidos pela COVID-19 foram muito além das alas hospitalares, a execução da atenção farmacêutica nas farmácias públicas e privadas foi essencial para o uso racional de medicações diante do desespero e caos gerados pelo não conhecimento de uma terapia específica e a disseminação rápida de muitas informações com ou sem respaldo científico.

Por fim, conforme Silva MJS, et al., (2020), a atuação dos farmacêuticos na pandemia causada pela COVID-19 foi essencial, tanto no fornecimento e controle dos estoques dos medicamentos e materiais médico-hospitalares, como em suas atividades assistenciais clínicas, lidando com o uso seguro e racional de medicamentos, prevenindo erros relacionados a medicamentos.

Portando em 2020 a palavras COVID-19, Pandemia, Recursos escassos se tornaram desafios reais que se apresentaram nessa importante fase de residência. Uma farmácia satélite, que atendesse diversos setores que compunham esse quadro de atendimento ao COVID-19, parecia bastante racional. Interagir com a equipe multiprofissional, elaborando protocolos em conjunto para uso seguro e racional de medicamentos, visto que estes estavam escassos, mostrou-se eficaz. Buscou-se também um trabalho de padronização de medicamentos, avaliação de estabilidade, aprazamentos simultâneos de mesmas drogas, o que muito colaborou nesse processo. 


\section{REFERÊNCIAS}

1. BARLOW A, et al. Review of Emerging Pharmacotherapy for the Treatment of Coronavirus Disease 2019. Pharmacotherapy: The Journal of Human Pharmacology and Drug Therapy, 2020. 40(5) 416-437.

2. BERNARDI EAT, et al. Implantação da avaliação farmacêutica da prescrição médica e as ações de farmácia clínica em um hospital oncológico do sul do Brasil. Revista Espaço Para A Saúde, 2014: 15(2); 29-36.

3. BRASIL. Diretrizes para Diagnóstico e Tratamento da COVID-19: Versão 1. Brasília: Ministério da Saúde, 2020. Disponivel em: https://pncq.org.br/uploads/2020-1/Diretriz-Covid19-v4-07-05.20h05m.pdf. Acessado em: 08 de janeiro de 2021.

4. CARDINAL L e FERNANDES C. Intervenção Farmacêutica no Processo de Validação da Prescrição Médica. Rev. Bras. Farm. Hosp. Serv. Saúde, 2014: 5(2); 14-19.

5. CASSANO AO e AREDAS CA. A flexibilização de requisitos brasileiros de Boas Práticas

6. CONSELHO FEDERAL DE FARMÁCIA. Resolução № 585 - Regulamenta as atribuições clínicas do farmacêutico e dá outras providências. Publicada no DOU. 29 de agosto de 2013 . Disponível em: https://www.cff.org.br/userfiles/file/02resolucoes/585.pdf. Acessado em: 03 de janeiro de 2021.

7. CONSORTIUM, Who Solidarity Trial (org.). Repurposed Antiviral Drugs for Covid-19 — Interim WHO Solidarity Trial Results. New England Journal Of Medicine, 2020; 1-15.

8. DANTAS FA. A descentralização da farmácia do pronto socorro: análise da distribuição de 4 kits de materiais. TCC (Graduação Curso de Enfermagem) - Faculdade de Medicina da Universidade Federalde Uberlândia, Uberlândia, 2019: 35.

9. de Fabricação durante a pandemia da COVID-19 sob uma perspectiva comparada. Vigil. sanit. Debate, 2020: 8(3):4451.

10. GIL MG e AMO CV. Hospital Pharmacist experience in the Intensive Care Unit: Plan COVID. Farmacia Hospitalaria, 2020: 44(1); 32-35.

11. LLAMAZARES CMF, BRIZ EL. Retos del farmacéutico de hospital en la evaluación de la evidencia científica y su incorporación a los protocolos farmacoterapéuticos a través de las comisiones en tiempos de COVID-19. Farm Hosp, 2020; 24-7.

12. MCCONACHIE S, et al. Surviving the surge: Evaluation of early impact of COVID-19 on inpatient pharmacy services at a community teaching hospital. American Journal of Health-System Pharmacy, 2020: 77; 1994-2002.

13. MONTEIRO FFC, et al. O papel da assistência farmacêutica no tratamento de pacientes com COVID-19 em hospital de referência no município de Fortaleza. Revista de Casos e Consultoria, 2020: 11(1).

14. PELENTIR M, et al. Importância da assistência e atenção farmacêutica no ambiente hospitalar. Rev. Ciência e Tecnologia, 2015: 1(1): 20-28.

15. PILAU R, et al. Atuação do Farmaceutico Clinico em Unidade de Terapia Intensiva Adulto: Uma Revisão da Literatura. Rev. Bras. Farm. Hosp. Serv. Saúde,2014: 5(1): 19-24.

16. SILVA BC, OLIVEIRA JV. A importância da atuação permanente do farmacêutico na equipe multidisciplinar da UTI em benefício da saúde do paciente e redução de custos para um hospital no município de Imperatriz-MA. TCC (Graduação Curso de Farmácia) - Faculdade Imperatriz, Imperatriz, 2012: 15.

17. SILVA LMC, ARAUJO JL. Clinical and community pharmacist's role in the COVID-19 pandemic. Research, Society and Development, 2020: 9(7); 1-14.

18. SILVA MJS, et al. Nota Técnica da Sociedade Brasileira de Farmacêuticos em Oncologia sobre Assistência Farmacêutica em Oncologia frente à Pandemia de Covid-19. Revista Brasileira de Cancerologia, 2020; 66.

19. SOCORRO FHOS, et al. As funções da equipe pluridisciplinar no cuidado da covid-19. Braz. J. Hea. Rev,2020: 3(5); 17577-12591.

20. WU Y, et al. The outbreak of COVID-19. Journal Of The Chinese Medical Association, 2020: 83(3); $217-220$.

21. VALLE MCD, et al. Contribuições da Farmácia, Fisioterapia e Psicologia a pacientes com COVID-19 em Unidades de Terapia Intensiva. Health Residencies Journal, 2020: 1(5). 